\title{
Utilisation of eye and skin care, and social services among persons with albinism in Ulundi, KwaZulu-Natal, South Africa
}

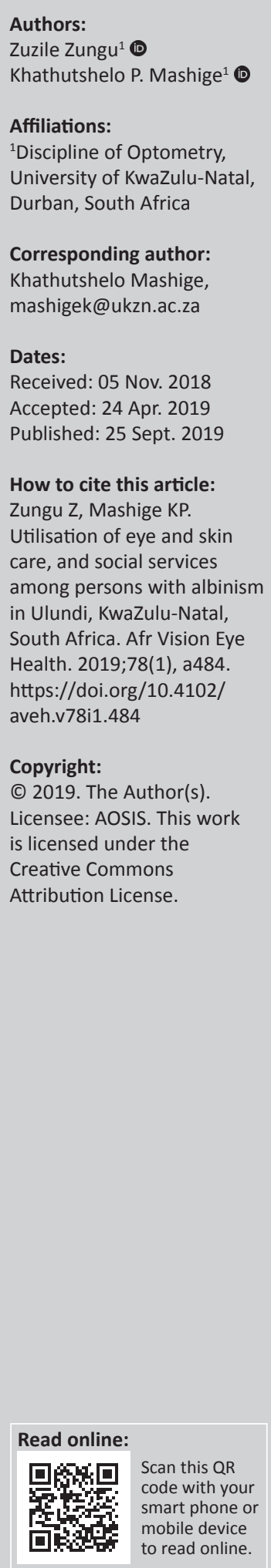

Background: Knowledge of the utilisation of eye and skin care, and social services among persons with albinism is essential for planning intervention strategies for this group.

Aim: The aim of this study was to determine the utilisation of eye and skin care, and social services among persons with albinism in the Ulundi Municipality of KwaZulu-Natal Province, South Africa.

Setting: The Ulundi Municipality of KwaZulu-Natal, South Africa.

Methods: The cross-sectional survey used a questionnaire to collect data from 21 participants living with albinism. Semi-structured face-to-face interviews were conducted in small peer groups.

Results: The participants ( $n=21$; males 8 and females 13 ) were aged 4 to 60 years (mean $=24.5 \pm$ 17.9 years). All participants reported that they had their eyes tested within the last two years by an optometrist and none by an ophthalmologist. Almost half (42.9\%) had never used a low vision device, this being because of their non-availability (44.4\%), financial constraints (33.3\%) and lack of or poor awareness (22.2\%). Participants reported wearing a wide-brimmed hat $(47.6 \%)$, a combination of wide-brimmed-hats and sunglasses $(33.3 \%)$ for eye protection and $19 \%$ reported using neither. All the participants reported that they used sunscreen with sun protection factor and $90.5 \%$ reported using long-sleeved shirts for skin protection. The majority (81\%) of participants reported receiving the South African Social Security Agency (SASSA) social (visual disability) grant, with $85.7 \%$ knowing where the SASSA offices were located.

Conclusion: Despite the poor use of low vision aids, the level of utilisation of eye and skin care, and other social services among participants was good.

Keywords: albinism; utilisation; eye care services; eye protection; skin care; social services.

\section{Introduction}

Oculocutaneous albinism (OCA) is a congenital condition characterised by a decrease in, or absence of, pigmentation of the hair, skin and eyes. ${ }^{1}$ This condition is the most prevalent type of albinism in Africa, and its diagnosis in a black population is clear and unequivocal. ${ }^{1}$ Oculocutaneous albinism presents with typical clinical features of pale, chalky, de-pigmented skin, sandy coloured hair and blue to hazel eyes. ${ }^{1}$ These phenotypic characteristics are typically responsible for the associated social stigmatisation and discrimination. ${ }^{1}$ Ocular problems are very common in albinism, and conditions such as photophobia (extreme sensitivity to light), myopia, nystagmus and strabismus develop because of a lack of retinal pigment. ${ }^{2}$ Hypopigmentation of the fundus occurs because of the reduction of melanin in the choroid and retinal pigment epithelial layer. ${ }^{2}$

Albinism has been reported ${ }^{3}$ to be a major cause of childhood visual impairment in the rural region of Limpopo Province, South Africa, where the frequency is 1:1500-1:1900 among the black population. ${ }^{4}$ Besides the vision impairment, exposure to ultraviolet radiation in this group induces skin damage and increases the risks of cancer. ${ }^{5}$ They are also highly susceptible to sunburn, blisters, solar elastosis and squamous cell carcinomas., ${ }^{5,6}$ It has been well documented that squamous cell carcinomas of the head and neck are the most common cutaneous tumours seen in African patients with albinism, while basal cell carcinomas occur less frequently. ${ }^{7}$ These cancers can significantly increase morbidity, with their treatment regimens generally being expensive. ${ }^{7}$ It is therefore important for persons with albinism to protect themselves from the sun at a young age to limit the development of skin damage. 
Many people living with albinism fall within the low vision category definition of visual acuity (VA) of less than $6 / 18$ but equal to or better than $3 / 60,{ }^{8}$ and are legally entitled to a social grant or visual disability grant from the government of South Africa. The social services disability grants represent a major policy response to issues of poverty among the disabled and children in South Africa. Dealing effectively with the health and social issues surrounding persons with albinism in resource-limited countries, such as South Africa, is a challenge. As there is a need to understand and address health and social factors for persons with albinism, this study aimed to investigate the utilisation of eye and skin care, and social services among persons with the condition in the Ulundi Municipality of KwaZulu-Natal Province, South Africa.

\section{Methods}

This was part of a larger study to obtain data on the prevalence and distribution of OCA among household heads from 28 wards of Ulundi, which were selected through a systematic sampling. Every sixth household was included in the study and 500 household heads were interviewed to determine the prevalence and distribution of OCA. Data on sociodemographics and utilisation of eye and skin care, and social services were collected from 21 persons identified with OCA. Personal observation at the researcher's optometry practice over the last 12 years suggests that there are many people with OCA in the Ulundi area of KwaZulu-Natal. Some families present at the practice with multiple siblings with the condition. It was therefore considered of interest to investigate the utilisation of eye and skin care, and social services among individuals with OCA because anecdotal evidence suggests that many with visual impairment because of albinism were not accessing these services. A survey was conducted of 500 households, with the study population consisting of the 21 persons living with albinism who were residents at the time of the study. For the four participants who were younger than 10 years old, their parents or caregivers responded on their behalf. Where the appropriate respondent was unavailable, an appointment was made to return at a convenient time and followed up three consecutive times. A questionnaire containing both closed and open-ended questions was used to collect data through face-to-face interviews.

Open-ended questions were asked to elicit detailed information and follow up their responses for verification. These included face-to-face interviews in addition to fieldnote observations of the behaviours of participants. The environmental conditions were qualitatively noted to give context and understanding that interviews alone would not have provided. The interviews were conducted at the participants' homes, which enabled the trained community development workers to observe the participants' domestic circumstances and compare this with their responses. Besides obtaining the socio-demographic information, questions were asked on their utilisation of eye and skin care, and social services. The questionnaire consisted of four sections: sociodemographic data; utilisation of eye care services; utilisation of skin care and protection; and utilisation of social services.
All returned questionnaires were reviewed and checked for accuracy and completeness of all variables. The questionnaires were pre-coded prior to data capturing to reduce errors that may have resulted from incorrect data entry. The data were captured by a trained data capturer onto Epi-info 2000 and analysed using the Statistical Package for the Social Sciences (SPSS Inc., Chicago, IL). The data were analysed by a statistician from the University of KwaZulu-Natal. The data from closed-response questions were analysed descriptively and open-response questions were summarised.

\section{Ethical considerations}

Ethics clearance was obtained from the University of KwaZulu-Natal's Humanities and Social Science Research Ethics Committee. Ethical Clearance number: HSS/0100/012M. The key ethical considerations in this study were voluntary participation, informed consent, avoidance of harm, confidentiality and anonymity. Because we were dealing with a vulnerable group, we also sought the advice of local authorities when pilot-testing the study to minimise cultural sensitivities and difficulties associated with conducting a study of this nature.

\section{Results}

\section{Socio-demographic information}

There were 21 individuals with albinism, their ages ranging from 4 to 60 years (mean $=24.5 \pm 17.95$ years), of whom eight were males and 13 were females - the majority of whom were students (Table 1).

\section{Utilisation of eye care services}

All the participants reported that they had eye problems, with all suffering from difficulties seeing objects at distance, photophobia, painful eyes and difficult in walking on steps (Table 2). When asked what other eye problems they experienced, nystagmus was reported as a general problem. For instance, one participant mentioned that 'My eyes move from side to side (referring to nystagmus) and I have seen these eye movements in other people with albinism'. They had all had their eyes tested at some point in their lives; $19(90.5 \%)$ had been examined by private optometrists and

TABLE 1: Socio-demographic profile of the participants.

\begin{tabular}{llcc}
\hline Variable & Options & No. & $\%$ \\
\hline Gender & Male & 8 & 38.1 \\
& Female & 13 & 61.9 \\
Age categories (years) & $<5$ & 1 & 4.8 \\
& $5-10$ & 3 & 14.3 \\
& $10-20$ & 4 & 19.0 \\
& $20-30$ & 10 & 47.6 \\
& $30-40$ & 1 & 4.8 \\
& $>40$ & 2 & 9.5 \\
Employment and & Students & 11 & 52.4 \\
occupation status & Unemployed & 6 & 28.6 \\
& Employed & 2 & 9.5 \\
& Crèche/Kindergarden & 1 & 4.8 \\
& Pensioner & 1 & 4.8 \\
\hline
\end{tabular}

No., number. 
TABLE 2: Utilisation of eye care services.

\begin{tabular}{llcc}
\hline Variable & Options & No. & \% \\
\hline Eye problems & Distance difficulties & 21 & 100 \\
& Painful eyes & 21 & 100 \\
& Photophobia & 21 & 100 \\
& Step walking & 21 & 100 \\
Time of last eye & 6 months ago & 4 & 19 \\
examination & 1 year ago & 8 & 38.1 \\
& 2 years ago & 9 & 42.9 \\
Examined by an & Yes & 0 & 0 \\
ophthalmologist & No & 21 & 100 \\
Spectacles worn & Yes & 19 & 90.5 \\
& No & 2 & 9.5 \\
$\begin{array}{l}\text { Use of low vision } \\
\text { devices }\end{array}$ & Yes & 12 & 57.1 \\
$\begin{array}{l}\text { Reasons for not using } \\
\text { visual aids }\end{array}$ & No & 9 & 42.9 \\
& Havancial constraints & 3 & 33.3 \\
& No available eye centre & 2 & 22.2 \\
$\begin{array}{l}\text { Use of eye care } \\
\text { products and } \\
\text { protection methods }\end{array}$ & Wide-brimmed hats & 4 & 44.4 \\
& Combination of wide-brimmed & 7 & 47.6 \\
& hats and sunglasses & 33.3 \\
\hline & Neither wide-brimmed hats nor & 4 & 19 \\
\hline
\end{tabular}

two $(9.5 \%)$ were tested in government hospitals. Despite the reported difficulties with distance vision, sensitivity to light and difficulty in walking on steps, nine (42.9\%) had their eyes tested two years prior to the study (Table 2).

Of the 21 participants, 19 (90.5\%) were wearing spectacles and none had been examined by an ophthalmologist, which is a general recommendation for persons with albinism. Twelve participants $(57.1 \%)$ reported using or having used low vision devices. Eye protection was reported to be used by 17 (81\%) participants, despite 21 (100\%) reporting photophobia, with $10(47.6 \%)$ using hats and seven (33.3\%) using a combination of wide-brimmed hats and sunglasses.

\section{Utilisation of skin care and protection}

Fifteen $(71.4 \%)$ participants in the study reported having problems with their skin. Although not mutually exclusive, conditions such as blisters, sores and sunburn were the most frequently reported skin problems. If more than one condition occurred simultaneously, the primary cause was determined considering the severity of the condition as to which contributed more. Others $(28.6 \%)$ indicated not having any problems with their skin. All the participants reported using sunscreens and 19 (90.5\%) indicated that they used protective wear such as long-sleeve shirts for protection against sunlight (Table 3). Of the 21 (100\%) participants who used sunscreen, $16(76.2 \%)$ participants indicated that they used a sun protection factor (SPF) of over 30, five (23.8\%) did not know, despite the fact that they were using sunscreen with SPF.

Participants indicated that SPF was provided by the hospital free of charge; however, when these suppliers ran out of stock, they had to buy them. Most participants reported ease with the application of sunscreen in terms of acceptability; however, there were some who were not sure about the exact purpose and function of the sunscreen lotion. For instance,
TABLE 3: Utilisation of skin protection.

\begin{tabular}{llcc}
\hline Variable & Options & No. & $\mathbf{\%}$ \\
\hline Skin problems & Yes & 15 & 71.4 \\
& No & 6 & 28.6 \\
\multirow{2}{*}{ Types of skin conditions } & Blisters & 7 & 33.3 \\
& Sores & 5 & 23.8 \\
& Sunburn & 3 & 14.3 \\
\multirow{2}{*}{ Skin protection methods } & Long sleeves & 19 & 90.5 \\
& Sunscreen with SPF & 21 & 100 \\
\hline
\end{tabular}

TABLE 4: Utilisation of social grants for visual disability.

\begin{tabular}{llcc}
\hline Variable & Options & No & $\mathbf{\%}$ \\
\hline Social grant recipient for visual disability & Yes & 17 & 81 \\
& No & 4 & 19 \\
Renewal time for social grants & Every 6 months & 5 & 23.8 \\
& Every year & 1 & 4.8 \\
& Every 2 years & 9 & 42.9 \\
& Every 3 years & 2 & 9.5 \\
& Every 5 years & 4 & 19 \\
Location of SASSA offices known & Yes & 18 & 85.7 \\
& No & 3 & 14.3 \\
\hline
\end{tabular}

SASSA, South African Social Security Agency.

some participants indicated that 'We use sunscreens on our faces, necks and arms but not on our hands and fingers.'

\section{Utilisation of social grant for visual disability}

Seventeen $(81 \%)$ of the 21 participants reported that they received the South African Social Security Agency (SASSA) social (visual disability) grant (Table 4). Five (23.8\%) participants indicated that they renewed the grant every six months and nine (42.9\%) participants every two years (Table 4). The renewals were done as per the stipulation of SASSA and at the discretion of the district surgeon, who had to make recommendations for the grant to be provided. Twelve $(57.1 \%)$ participants reported receiving good treatment from the government officials when accessing the social grant services, but nine (42.9\%) participants indicated the contrary. Eighteen $(85.7 \%)$ participants knew the locations of their regional SASSA offices and three (14.3\%) participants did not know where the SASSA offices were located (Table 4).

\section{Discussion}

The observed age characteristic is similar to the findings of previous African reports by Kromberg et al. ${ }^{6}$ in Soweto (South Africa), Okoro ${ }^{9}$ in Nigeria, Hong et al. ${ }^{10}$ in Tanzania and a European report by Collins and Silver. ${ }^{11}$ Although pro-male gender distributions have been previously reported, ${ }^{11,12,13,14}$ the present finding found a higher distribution of albinism among females. For example, the participants in Udeh et al. ${ }^{14}$ study comprised more males than females, with an age range of 6-60 years.

Many participants reported all the classical ocular clinical presentations of albinism such as reduced vision, nystagmus and photophobia. These ocular features of OCA are caused by a reduction in pigment at the fovea. ${ }^{10}$ All the participants indicated that they have had their eyes tested at some point in their lives, and $90.5 \%$ were wearing spectacle corrections. This 
suggests that refractive anomalies still play a significant role in most participants, and spectacle correction improved vision and reduced the number of participants with visual impairment. Eye care practitioners and government personnel involved in albino-specific vision care services should be aware of these and provide resources to attend to the correction of refractive anomalies. Jhetam and Mashige ${ }^{15}$ reported that following optical correction, VA significantly improved from a range of 0.50-1.40 $\log$ MAR to a range of 0.5-1.06 logMAR for distance $(p<0.05)$ and from a range of $0.40-1.30 \log$ MAR to a range of $0.30-1.08 \log$ MAR for near $(p<0.05)$. Similarly, Udeh et al. ${ }^{14}$ reported that spectacle correction significantly improved the overall mean distance and near visual acuities of patients with albinism in their study.

None reported having seen an ophthalmologist for their condition, possibly because of the lack of ophthalmological services in the study area. A recent report indicates that the Ulundi area has no ophthalmologist and the nearest ophthalmologist is situated $200 \mathrm{~km}$ from the study area. ${ }^{16}$ In addition, the cost implications of transport and services of the ophthalmologists in the nearest area could have influenced this result. These results suggest the need for ophthalmological services to be made available to these communities particularly those with albinism as management protocols for this condition require a comprehensive multi-disciplinary approach, including ophthalmologists, optometrists, psychologists and so on.

Despite the reported high compliance to the clinical recommendations of an eye test at least once every two years (Table 2), not all participants were using low vision aids. Nonavailability, financial constraints and lack of awareness were the participants' main barriers to accessing low vision devices (Table 2). Udeh et al. ${ }^{14}$ reported poor awareness (have never heard about low vision assessment or low vision aids) in 136 $(89.0 \%)$ participants as the main reason for not utilising vision care services. Other reasons reported by the authors ${ }^{14}$ included 'no available centre' in $13(8.5 \%)$ and 'financial constraints' in $4(2.6 \%)$ participants. There is a need for the government to make low vision devices available and affordable as well as to create awareness of this service. In addition, when there are resource constraints, non-governmental organisations, such as the Brien Holden Vision Institute, can be alternatives as they can work with government to provide affordable eye care services and low vision devices to this group.

Wearing sunglasses, hats, long-sleeve clothing and using sunscreen lotions are simple and effective photo-protective measures. This study found that a significant proportion of participants used eye and skin protective measures. This is good as persons living with albinism are sensitive to the sun and are prone to skin disease - secondary to exposure to ultraviolet from sunlight. Gaigher et al. ${ }^{17}$ also reported that the use of protective measures (such as umbrellas) against sunlight were important for persons living with albinism. In South Africa, persons living with albinism are provided with generic SPF-15 sunscreens free of charge by the government. They are taught how to apply sunscreen regularly and to wear sun protective clothing. SPF-15 cream is effective in blocking ultraviolet $\mathrm{B}(\mathrm{UVB})$ radiation, and research suggests that a SPF-30 increases the effectiveness in blocking UVB only marginally from $93 \%$ to $96 \% .{ }^{18}$ However, participants raised concerns about sometimes having to buy their own sunscreens when there was insufficient supply of sunscreen lotions from the hospital. This concern possibly emanates from the prohibitive cost and access to sunscreen lotions when the hospitals had run out of stock. Lund and Taylor ${ }^{19}$ also reported that 'government sponsored sunscreen preparations were only provided if actively sought, involving time consuming trips to regional hospitals, with inadequate availability and insufficient supply'.

Although most participants used sun protective clothing, it is still important to apply sunscreen to the parts of the body that may not be adequately covered and left exposed to the sun. In OCA, the consequent reduction in cutaneous melanin increases the risk of non-melanoma skin cancer. ${ }^{20,21,22}$ Participants in this study took measures to reduce their sun exposure and wore hats, yet some did not fully understand the purpose of applying sunscreen. McBride and Leppard ${ }^{23}$ also concluded in a study conducted in Tanzania that some patients did not understand the purpose of applying sunscreen, with $10 \%$ also applying it at night. The authors suggested that these findings highlighted the importance of evaluating health promotional messages.

Most of the participants were on the visual disability grant. However, they reported inconsistencies with regard to the renewal of the disability grant that varied from six months up to five years. These inconsistencies could be an indication of the reported challenges (including corruption) that the SASSA faces. The Department of Women, Children and People with Disabilities (DWCPD) in partnership with the Albinism Society of South Africa (ASSA) have also acknowledged these challenges. ${ }^{24}$ In addition, other challenges acknowledged by DWCPD and ASSA include the backlog of non-recognition, discrimination, stigmatisation, prejudice and their isolation even within government departments.

\section{Conclusion}

Participants in this study tested their eyes regularly and many were wearing spectacle correction. Participants wore sunglasses, hats and long-sleeved shirts and used sunscreens to protect their eyes and skin. However, the quantity and reliability of supply of sunscreens from the government hospitals should be improved. Besides the low utilisation of low vision aids, many medical (eye and skin care) and social services needs of persons with albinism in the Ulundi area were met.

\section{Acknowledgements}

Khathutshelo Percy Mashige is a University of KwaZuluNatal (UKZN) Developing Research Innovation, Localisation and Leadership in South Africa (DRILL) fellow. DRILL, is a NIH D43 grant (D43TW010131) awarded to UKZN in 2015 to support a research training and induction programme for early career academics. The content is solely the responsibility 
of the authors and does not necessarily represent the official views of DRILL and the National Institute of Health. The authors thank Prof. O.A. Oduntan for reviewing this article and giving valuable comments, and also thank all the participants who took part in this study.

\section{Competing interests}

The authors declare that they have no financial or personal relationships that may have inappropriately influenced them in writing this article.

\section{Authors' contributions}

Z.Z. collected the data and wrote the manuscript as part of her master's thesis under the supervision of K.P.M. Review of the initial draft, supervision of revisions and approval of the article were performed by K.P.M.

\section{Funding information}

This research received no specific grant from any funding agency in the public, commercial or not-for-profit sectors.

\section{Data availability statement}

Data sharing is not applicable to this article.

\section{Disclaimer}

The views and opinions expressed in this article are those of the authors and do not necessarily reflect the official policy or position of any affiliated agency of the authors'.

\section{References}

1. Lund PM. Oculocutaneous albinism in Southern Africa: Population structure health and genetic care. Annals Hum Biol. 2005:32(2):168-173. https://doi. org/10.1080/03014460500075423

2. Summers CG. Albinism: Classification, clinical characteristics, and recent findings Optom Vis Sci. 2009;86(6):659-662. https://doi.org/10.1097/OPX.0b013e3181 a5254c
3. Venter PA, Christianson AL, Hutamo CM, et al. Congenital anomalies in rural black South African neonates - A silent epidemic? S Afr Med J. 1995;85(1):15-20.

4. Lund $P$, Maluleke $T$, Gaigher I, et al. Oculocutaneous albinism in a rural community of South Africa: A population genetic study. Annals Hum Biol. 2007;34(4): 493-497. https://doi.org/10.1080/03014460701401261

5. Bothwell JE, Abs T. Pigmented skin lesions in tyrosine-positive oculocutaneous albinos: A study in black South Africans. Inter J Dermatol. 1997;36(11):831-836.

6. Kromberg JG, Castle D, Zwane EM, et al. Albinism and skin cancer in Southern Africa. Clin Genet. 1989;36(1):43-52.

7. Yakubu A, Mabogunje OA. Skin cancer in African albinos. Acta Oncologica. 1993;32(6):621-622.

8. Sacharowitz HS. Visual impairment in South Africa: Achievements and challenges. S Afr Optom. 2005;64(4):139-149. https://doi.org/10.4102/aveh.v64i4.239

9. Okoro AN. Albinism in Nigeria. Br J Dermatol. 1975;92(5):485-492.

10. Hong ES, Zeeb $\mathrm{H}$, Repacholl MH. Albinism in Africa as a public health issue. BMC Pub Health. 2006;6:212. https://doi.org/10.1186/1471-2458-6-212

11. Collins B, Silver J. Recent experiences in management of visual impairment in albinism. Ophthal Genet. 1990;11(3):225-228.

12. Oduntan AO, Raliavhegwa M, Lund PM. Vision status of children with oculocutaneous albinism. Ophthal Physiol Opt. 2002;22(6):572-573. https://doi. org/10.1046/j.1475-1313.2002.00086_3.x

13. Mohamed AF, El-Sayed NS, Seifeldin NS. Clinico-epidemiologic features of oculocutaneous albinism in northeast section of Cairo, Egypt. Egypt J Med Hum Genet. 2010;11(2):167-172. https://doi.org/10.1016/j.ejmhg.2010.03.001

14. Udeh NN, Eze BI, Onwubiko SN, et al. Oculocutaneous albinism: Identifying and overcoming barriers to vision care in a Nigerian population. J Comm Health 2013;39(3):508-513. https://doi.org/10.1007/s10900-013-9787-5

15. Jhetam S, Mashige KP. Ocular findings and vision status of learners with oculocutaneous albinism. Afr Vision Eye Health. 2019;78(1):a466. https://doi. org/10.4102/aveh.v78i1.466

16. Medical network [homepage on the Internet]. [cited 2017 Dec 22]. Available from: https://www.medicalnetwork.co.za

17. Gaigher R, Lund P, Makuya E. A sociological study of children with albinism at a special school in the Limpopo province. Curationis. 2002;25(4):4-11.

18. Preston D, Stern R. Nonmelanoma cancers of the skin. New Eng J Med. 1992;327(23):1649-1662. https://doi.org/10.1056/NEJM199212033272307

19. Lund PM, Taylor JS. Lack of adequate sun protection for children with oculocutaneous albinism in South Africa. BMC Public Health. 2008;8:225. https:// doi.org/10.1186/1471-2458-8-225

20. Oduntan AO. Prevalence and causes of low vision and blindness worldwide. S Afr Optom. 2005;64(2):44-54. https://doi.org/10.4102/aveh.v64i2.214

21. Oetting WS, King RA. Molecular basis of albinism: Mutations and polymorphisms of pigmentation genes associated with albinism. Hum Mutation. 1999;13(2) 99-115. https://doi.org/10.1002/(SICI)1098-1004(1999)13:2<99::AID-HUMU2>3. $0 . \mathrm{CO} ; 2-\mathrm{C}$

22. Karen G, Ek J, Karen B. Oculocutaneous albinism. Orphanet J Rare Diseases. 2007;2:43. https://doi.org/10.1186/1750-1172-2-43

23. McBride S, Leppard B. Attitudes and beliefs of an albino population toward sun avoidance. Advice and services provided by an outreach albino clinic in Tanzania. Arch Dermatol. 2002;138(5):629-632.

24. Department of Social Development. The social and economic impact of South Africa's Social Security System. Summary Report. Pretoria: Economic Policy Research Institute; 2004. 